\title{
Análisis de los Principales Problemas para la Impartición de Clases en el Posgrado desde un Enfoque Interdisciplinario
}

\section{Analysis of the Main Problems for Teaching of Classes in the Postgraduate Course from an Interdisciplinary Approach}

\author{
Ernesto Bolaños-Rodríguez ${ }^{a}$ Evangelina Lezama-León ${ }^{a}$, Gaby Y. Vega-Cano ${ }^{a}$
}

\begin{abstract}
:
The paper analyzes the main difficulties that arise in the delivery of classes in the postgraduate course, particularly in specialties, masters and doctorates from an interdisciplinary perspective. The research is carried out using the systems approach applied to the teaching-learning process where the didactic categories are conceived: objectives, contents, methods and strategies, forms of organization, resources and evaluation, as well as their interrelation. The main conclusions show that if there is no domain of the disciplines that interact to address an object of study from different reference frames and a "favorable environment" the expected impact will not be achieved.
\end{abstract}

Keywords:

Interdisciplinary, postgraduate, systems, didactic and teaching-learning process.

\section{Resumen:}

El trabajo analiza las principales dificultades que se presentan en la impartición de clases en el posgrado, particularmente en especialidades, maestrías y doctorados desde una perspectiva interdisciplinaria. La investigación se lleva a cabo utilizando el enfoque de sistemas aplicado al proceso de enseñanza-aprendizaje donde se conciben las categorías didácticas: objetivos, contenidos, métodos y estrategias, formas de organización, recursos y evaluación, así como su interrelación. Las principales conclusiones evidencian que si no existe un dominio de las disciplinas que interactúan para abordar un objeto de estudio desde diferentes marcos de referencias y perspectivas, así como un "ambiente favorable" no se logrará el impacto esperado.

\section{Palabras Clave:}

Interdisciplina, posgrado, sistemas, didáctica y proceso de enseñanza-aprendizaje.

\section{Introducción}

Es muy común que en diversos programas de posgrados: especialidades, maestrías y doctorados se aborden fenómenos, hechos, acontecimientos, contenidos y principios de un mismo objeto de estudio desde diferentes perspectivas y marcos de referencias, que si fueran abordados desde una sola disciplina no conllevaría a lograr los alcances en las soluciones de los retos y desafíos que se presentan en el mundo de hoy.

Para Morin (2006), se necesita que el ser humano adquiera conocimientos sin divisiones ni compartimentos, de forma tal que se respete también lo individual y lo singular, considerando su interrelación con el contexto y el todo. 1
Para los hombres de ciencia de hoy y del futuro se requiere no sólo una sólida formación técnica, sino también humanista y de responsabilidad social que vaya encaminada a salvaguardar la vida en la tierra amenazada por severas crisis sociales, ambientales, políticas, económicas, de escasez de recursos, entre otras.

La generación de los futuros hombres de ciencias demanda una preparación para la vida donde la universidad sea el vehículo que conduzca a un modo de actuación que prepondere todos los saberes de manera holística y que no deje de lado la importancia de éstos en la convivencia humana.

\footnotetext{
aUniversidad Autónoma del Estado de Hidalgo, Escuela Superior de Tizayuca,Email: ebolanos@uaeh.edu.mx 
Es por ello, que juega un papel fundamental en la educación posgraduada contemporánea evitar la racionalización extrema y que exista un compromiso con la complejidad que permita como resultado de las investigaciones que se llevan a cabo en las tesis, proyectos terminales y actividades académicas productos que estén pensados en función de la sociedad a través de acciones concretas para solucionar problemas.

Por consiguiente, el docente en los programas de estudios de posgrado tiene la difícil misión de convertirse en guía y facilitador del aprendizaje de profesionistas de diferentes áreas del conocimiento y en muchas ocasiones imparten sus clases desde un enfoque interdisciplinario y con ello se enfrenta a múltiples situaciones para los cuales no está capacitado. 2

Es por ello, que el perfil docente del catedrático que imparte clases en este nivel de enseñanza debe considerar la tutoría como una investigación que enseña y una enseñanza que estimula la investigación.

Además, debe ser un creador de escenarios educativos donde los estudiantes se involucren en la didáctica y a través de ésta logren potenciar su conocimiento. 3

A partir de todo lo anteriormente expuesto el objetivo del trabajo se centra en analizar los principales problemas que enfrenta el catedrático de posgrado para abordar contenidos desde una perspectiva interdisciplinaria a través del uso del enfoque de sistema que permita mediante un proceso de mejoramiento continuo lograr el aprendizaje esperado en el estudiante.

\section{Referentes teóricos}

\section{Aspectos Esenciales en la Formación de los Estudiantes del Posgrado}

El hombre adquiere conocimientos en la medida que se relaciona e interactúa con fenómenos naturales y sociales, pero trascienden en el individuo en la medida que tienen sentido y significado, lo cual le posibilita lograr aprendizajes que perduren en el tiempo.

La formación posgraduada actual requiere el dominio e integración de saberes (conocimientos, habilidades, hábitos, aptitudes, destrezas, actitudes y valores) que le permita al estudiante alcanzar el nivel de desarrollo de las competencias y que por ende lo convierta en especialista de alto nivel apto para afrontar los vertiginosos avances científicos y tecnológicos y los cambios que se avizoran a corto y mediano plazo. 4
A partir de estas exigencias es que resulta propicio el diseño curricular a nivel de posgrado, de manera que sea flexible, pertinente y factible, lo cual posibilite la movilidad estudiantil de un programa de estudios a otro y con ello se fomente la interdisciplina como una filosofía de trabajo. 5

De esta manera es esencial, en la preparación del investigador, contar con un conocimiento preciso de la ciencia de estudio en particular, y su vínculo con otras ramas que le permitan una cosmovisión del hecho concreto a investigar. Por lo que resulta vital formar un pensamiento en este tipo de estudiante que contribuya a la asimilación de la estructura de las etapas del proceso de investigación: cuestionarse el problema, cómo va a presentar su diseño de investigación, de qué forma va interpretar los resultados que lo conlleve a las conclusiones y los trabajos a futuro.

También es imprescindible desarrollar las capacidades de análisis y síntesis, ellas se deben percibir como pares dialécticos, en la que el análisis no puede existir sin la síntesis y viceversa. El análisis permite estudiar el comportamiento de cada una de las partes, así como definir los elementos y aspectos que ejercen una influencia decisiva en las otras partes del objeto de investigación y determinan su comportamiento.

Sobre la base de la generalización de algunas características definidas como resultado del análisis, la síntesis teórica lleva a la formulación de leyes, teorías e hipótesis que explican la conducta del objeto de investigación en el momento actual, su comportamiento futuro, así como favorece el poder predecir su desarrollo, en condiciones específicas. 6

Algunas características que deben distinguir a un docente de nivel de posgrado son las relacionadas con la competitividad investigativa, de análisis y reflexión en cuanto al propio ejercicio educativo y profesional, el conocimiento y apropiación de las TIC, como herramienta de apoyo, implicación en las nuevas metodologías de didáctica educativa que permitan efectividad en el currículo. 7

Es esencial concebir a la educación posgraduada con carácter innovador a un docente con las competencias profesionales establecidas y que vayan acorde a las exigencias y requerimientos de cada programa. 8

Agazzi (2001) propone las siguientes orientaciones metodológicas para que desde la docencia el estudiante pueda realizar investigación interdisciplinar, 9, entre las que se destacan: 
- La investigación interdisciplinar debe partir de la individualización del problema y de los aspectos del mismo que requieren del enfoque de disciplinas bien individualizadas.

- Establecer claramente los distintos criterios disciplinares para abordar los datos.

- Explicitar el marco teórico de las disciplinas, sin cuestionar sus supuestos.

- Definir los conceptos empleados por las distintas disciplinas involucradas, relacionándolos con su marco conceptual y su metodología de acceder a los datos.

- Aceptar la autonomía de los procedimientos lógicos de las disciplinas.

Otro elemento a considerar para este tipo de alumno son las habilidades de expresión oral y escrita en lenguaje científico técnico, porque éste debe comunicarse de forma tal que no genere ambigüedades, sea claro, preciso, coherente, así como redacte de forma impersonal y domine las normas para dar créditos a los autores y no cometa alguna violación a la propiedad intelectual. Además, en su discurso de defensas de ideas, tópicos y problemáticas a resolver demuestre poder de síntesis, sea capaz de distinguir entre lo esencial y lo complementario, delimite el alcance del tema tratado, respete los tiempos establecidos y logre inducir las posibles preguntas de los que escuchan su exposición. En resumen, debe facilitar la comprensión de los que evalúan o intentan comprender lo realizado.

\section{Sistema Didáctico en la Educación Posgraduada}

La didáctica es la ciencia del proceso de enseñanzaaprendizaje (objeto de estudio) que se establece en una institución educativa y con la orientación de los docentes. 10

De ahí se deriva que un sistema didáctico está integrado por distintos componentes (categorías): objetivos, contenidos, métodos y estrategias, recursos de enseñanza, formas de organización de la actividad docente y evaluación. Todas estas categorías tienen una relación de subordinación con respecto al sistema didáctico en su conjunto y ellos entre sí deben tener una relación de coordinación.

Esto es válido para cualquier nivel de enseñanza desde la primaria hasta el posgrado, pero ésta última tiene sus particularidades, algunas de ellas se resaltan a continuación:

- Objetivos con un nivel de profundidad y alcance mayor con respecto a otros niveles de enseñanza.
-Contenidos (teóricos, procedimentales y actitudinales) encaminados al ejercicio de la profesión especializada 0 en los posgrados de investigación enfocados al aspecto científico.

- Métodos y estrategias con marcado carácter activo, donde predominen los: Estudio de Casos, Aprendizaje Basado en Problemas y Proyectos, así como los Contratos de Aprendizaje (ejemplo: el que se establece entre el director de la tesis y el aspirante a obtener un grado científico).

- Recursos de enseñanza: Que se adecuen a las necesidades de la actividad a desarrollar y desplieguen el ingenio y la creatividad del docente y el estudiante.

- Formas de organización: Deben trascender a los modos tradicionales, donde se creen espacios de interacción de la teoría con la práctica y les permita llegar a obtener productos innovadores. Además, fomente el trabajo colaborativo y cooperativo entre los participantes con formaciones disciplinares diversas y que puedan converger en abordar contenidos de manera interdisciplinaria.

- Evaluación: Visualizada como la forma de expresar y recopilar las evidencias con las que el alumno ha llegado alcanzar el nivel de competencia y el perfil de egreso declarado en el plan de estudio.

Estas categorías se interrelacionan y se coordinan de tal forma que permitan concebir al sistema como un todo, donde existen entradas, proceso, salidas $y$ retroalimentación.

La enseñanza del posgrado desde un enfoque interdisciplinario, vista como un sistema requiere de la integración de todas sus categorías y una actualización docente permanente, que permita a los profesores el desarrollo de habilidades didácticas y adquisición de conocimientos disciplinares para impartir con éxito este tipo de enseñanza.

Si esto no se produce, como en algunas ocasiones sucede, fundamentalmente derivado por la resistencia al cambio del catedrático, así como por la creencia de que como a él lo formaron de esa forma y tuvo éxitos es la más adecuada, entonces este tipo de profesor continuará impartiendo sus asignaturas desde el punto de vista de la ciencia en la que se ha formado.

\section{La Interdisciplina como Filosofía de Trabajo}

Existen varias definiciones de interdisciplina pero por su grado de vínculo teórico con el presente trabajo, se toma en cuenta la que plantea, que es la interacción real y efectiva entre dos o más disciplinas diferentes. Tal interacción puede pasar de la simple comunicación de 
ideas hasta la integración mutua de categorías y conceptos fundamentales, métodos de investigación, terminologías, procedimientos de enseñanza y otros aspectos derivados del desarrollo científico. En tal sentido, la interdisciplinariedad es una práctica, a menudo crítica y revolucionaria, que contribuye a cambiar reglas rutinarias de la comunicación educativa y formas obsoletas de socialización del conocimiento. 11

Debe concebirse la interdisciplina como una filosofía de trabajo que motiva a los estudiantes en los siguientes aspectos:

- Los enseña a transferir conocimientos.

- Involucra la comunidad como medio de aprendizaje.

- Los enseña a cómo analizar, explicar y aplicar conocimientos.

- Se basa en la competencia.

- Los enseña a tomar decisiones.

- Mejora la retención del conocimiento.

-Aprenden a trabajar cooperativamente con los demás.

- Le dan sentido y ven el valor de la experiencia educativa.

-Estimula la creatividad al enfrentarse a nuevas vías para apropiarse de los contenidos.

\section{Metodología}

Inicialmente a través del proceso de observación, un investigador debe hacer numerosas anotaciones $y$ bosquejarlas para tener un archivo permanente de sus ideas que más tarde le sirva como referencia. Se debe revisar periódicamente estas ideas y anotaciones previas para evitar pasar por alto un concepto importante. Las ideas y pensamientos son el recurso vital de la creatividad y no deben desecharse.

Esta actividad puede realizarse bajo los principios de un esquema general de sistema, el cual consiste en identificar la(s) entrada(s), el proceso (s), y la(s) salida (s).

Partiendo de la definición de Ackoff (2005) de lo que es un problema, se tiene que éste "es la desviación entre lo real y lo deseado", por tanto, la salida de cualquier sistema es lo que se desea lograr, es decir la situación deseada o lo que se quiere obtener del sistema. Previamente se deberá haber identificado lo que se tiene; esto es lo que en el momento del análisis serían las entradas. 12

La (s) entrada (s) es con lo que en realidad se tiene y esto quedaría como todo aquello con lo que se cuenta o tiene para iniciar algo y la(s) salida(s) es todo aquello que se quiere o desea funcione, trabaje, o se comporte el sistema en cuestión.
Para que esto sea real se tiene que trasladar este esquema dentro de un contexto, el cual indique donde se quiere que esto sea una realidad.

Las observaciones a este sistema deben ser lo más cuidadosas, detalladas y completas que se puedan para que reflejen las ideas claras del que visualiza el sistema durante el período en que su pensamiento sigue de cerca el sistema y, de esta forma, le ayude a retener su dominio. Las anotaciones breves o incompletas pueden ocasionar la pérdida de tiempo en la recuperación de conceptos olvidados o bien en pérdida de información.

Los comentarios generales pertenecientes a una idea o bosquejo deben ser parte del registro permanente.

De esta manera, resulta fácil concentrarse prematuramente en una solución particular del sistema antes de analizarlo completamente, más tarde, se dará cuenta de que su primera impresión no tenía el fundamento adecuado o éste era inexacto, debido al estudio incompleto de todos los factores, es decir, no se tiene una visualización sistémica del sistema.

La propuesta está fundamentada en el método de enfoque de sistema, el cual está dirigido a modelar el objeto mediante la determinación de sus componentes, así como las relaciones entre ellos, las que determinan por un lado la estructura del objeto y por otro su dinámica, su movimiento. 6

Las cualidades más importantes que debe cumplir un sistema son:

Componentes.

Estructura.

Principio de jerarquía.

Relaciones funcionales.

Componentes: Son aquellos elementos principales cuya interacción caracteriza cualitativamente el sistema.

Estructura del sistema: Es el modo de organización e interacción entre los componentes que lo integran y es consecuencia del orden que establecen las relaciones en que determinados componentes adquieren una mayor jerarquía y otros se subordinan.

Principio de jerarquía: Todo sistema presenta una estructura jerárquica, ya que está integrado por diferentes partes y componentes que pueden ser considerados a su vez como subsistemas. Los sistemas inferiores sirven de base a los superiores y éstos a su vez subordinan y condicionan a los inferiores. 
Las relaciones funcionales pueden ser de dos tipos: de coordinación y de subordinación.

Las relaciones funcionales de coordinación se presentan en la vinculación que debe existir entre los componentes del sistema de igual grado de jerarquía.

Las relaciones funcionales de subordinación se presentan entre componentes del sistema de diferentes grados de jerarquía, donde un conjunto de elementos conforma un subsistema que a la vez se subordina a un sistema mayor.

Para aplicarse al sistema didáctico todas las categorías que lo integran (objetivos, contenidos, métodos y estrategias, recursos de enseñanza, formas de organización de la actividad docente y la evaluación). Todos estos componentes tienen una relación de subordinación con respecto al sistema didáctico en su conjunto y ellos entre sí deben tener una relación de coordinación.

Independientemente que algunos de ellos se subordinen a otros, cada uno por separado constituye un subsistema de otro mayor, así los objetivos conforman un sistema que a su vez es un subsistema que se subordina al sistema didáctico.

\section{Análisis y discusión de resultados}

Al analizar los resultados obtenidos al aplicar el enfoque de sistema, se tiene como elementos de entrada los alumnos del posgrado (perfil de ingreso) que se matriculan a estudiar un determinado tema objeto de estudio en una asignatura o módulo de la especialidad, maestría y doctorado, el programa de la asignatura en cuestión y el docente que imparte la clase con una formación disciplinar base en función de un área del conocimiento, por ejemplo un experto en Estadística que da cátedra de esta materia.

Posteriormente el proceso de enseñanza aprendizaje que transforma las entradas a las salidas. Ese proceso de enseñanza aprendizaje se concibe desde la perspectiva interdisciplinaria donde el docente aborda, por ejemplo: Bioestadística para una Maestría en Ciencias de la Salud y tiene una visión desde la enfermería, la nutrición, la farmacia y la medicina, por citar un ejemplo.

Para las salidas se tienen en cuenta los problemas detectados en la impartición de clases en el posgrado desde la perspectiva interdisciplinaria.

Para este sistema didáctico es imprescindible la retroalimentación que se produce a la salida del proceso y que se vuelve analizar con los componentes de entradas y que pueden enriquecer y profundizar las respuestas a los problemas presentados.

También a través del enfoque de sistema se ven reflejadas la subordinación de unas categorías con otras del sistema didáctico, por ejemplo, los métodos y estrategias se subordinan a los contenidos de enseñanza y éstos a su vez a los objetivos.

Para ilustrar los elementos del párrafo anterior se toman en cuenta que el profesor establece métodos y estrategias activas como el estudio de casos de éxitos de empresas innovadoras en México, tratados de forma interdisciplinaria subordinados a los contenidos de características que distinguen a las organizaciones con ventajas competitivas y éstos a su vez se subordinan al objetivo de la sesión con relación a valorar a las empresas comercializadoras, manufactureras y de servicios que logran impulsar la innovación, además esta intencionalidad está subordinada al sistema didáctico en su conjunto que busca una plena correspondencia e interrelación con todos sus componentes didácticos.

Otro resultado desde el uso del enfoque de sistema se da a partir de utilizar métodos y estrategias útiles para la enseñanza interdisciplinaria, en el abordaje de temas actuales de interés comunitario y cercanos a la realidad de los estudiantes. A manera de ejemplo se puede citar el estudio de los biomateriales que se aplican en la elaboración de prótesis dentales para pacientes con diversos padecimientos. La población, con estos tipos de enfermedades necesita la reparación de alguna pieza dental y casi siempre existe un desconocimiento del tipo de material y las características que debe poseer. La síntesis de los biomateriales dentales, requiere de la participación de varias ciencias como la ingeniería biomédica, la química, la física, la biología, la ciencia de los materiales y la corrosión para dar solución integral a este problema.

Lo cual evidencia que la enseñanza desde un enfoque interdisciplinario requiere de materiales didácticos acordes al contexto, a la forma de organización de la actividad docente (taller, seminario, práctica de laboratorio), a las formas en que se va evaluar este contenido, a los métodos y estrategias empleados, a lo propios contenidos y los objetivos establecidos, que si se estudian como sistema indican que ninguna categoría didáctica es más importante que otra, sino que si una falla todo fracasa y no se logra el aprendizaje esperado.

Es válido aclarar de porque en un posgrado cualquiera existan estudiantes procedentes de diversas licenciaturas de variadas áreas de conocimientos no quiere decir que ya eso implique interdisciplina, porque si el docente aborda un objeto de estudio desde una única perspectiva y marco de referencia esa clase sigue teniendo un enfoque disciplinar. 
Por último, se resaltan los principales problemas detectados en la investigación para la impartición de clases en el posgrado desde una perspectiva interdisciplinaria utilizando el enfoque de sistemas, los cuales se detallan desde los dos actores educativos fundamentales del proceso de enseñanza-aprendizaje, el alumno y el docente.

\section{Para el estudiante:}

- Formas y hábitos de aprendizaje tradicionales que se arrastran desde los niveles básicos de educación.

- Dificultades para la interpretación de la información al no ingresar al posgrado con la capacidad de análisis y síntesis requerida para este nivel de estudio.

-Problemas en la comprensión lectora.

-Mal uso de las Tecnologías de la Información y Comunicaciones (TIC) con fines educativos al querer tener respuestas de forma instantáneas, sin un análisis profundo de las tareas o ejercicios recomendados por el docente para el estudio independiente.

-Dificultades en el desarrollo del ingenio y la creatividad para darle una solución a una situación problemática.

-Dificultades en la integración de equipos de trabajos, que además muestran resistencia al cambio por no querer realizar análisis desde una visión interdisciplinar.

\section{Para el catedrático:}

- La formación de los docentes es disciplinar y muestran resistencia al cambio por considerar que su asignatura y los métodos de ésta son los mejores dentro del plan de estudios y no dando oportunidad a las formas de abordarlo desde los enfoques de otras disciplinas.

- Los currículos de formación de muchos posgrados tienen un carácter profundamente arraigado a lo disciplinar.

-Existencia de pocos cursos, diplomados 0 capacitación para el enfoque interdisciplinario.

- La falta de experiencia en el trabajo interdisciplinar.

- Falta de tolerancia y de respetar el pensamiento divergente en cuerpos colegiados encargados de la revisión de tesis y demás tareas académicas solicitadas en los posgrados que además tienen la función de analizar y evaluar los mismos.

- No todos los catedráticos en un mismo programa de estudio (especialidad, maestría o doctorado) trabajan o fomentan el enfoque interdisciplinario.

\section{Conclusiones}

Las principales problemáticas para la impartición de las clases de posgrado desde una filosofía de trabajo interdisciplinario están identificadas desde la perspectiva de los dos actores educativos fundamentales: el estudiante y el docente, por lo que buscar las causas que lo provocan con vista a atacarlas generará al final del proceso de enseñanza-aprendizaje que los efectos serán eliminados o mitigados.

Para impartir docencia en un posgrado desde un enfoque interdisciplinario se necesita de un catedrático que sea capaz de mostrar una actitud de enfrentar al conocimiento desde varias aristas, sin creer que se está invadiendo el campo de acción de ninguna disciplina, ni que demuestre que una disciplina sea más importante que la otra u otras que interactúan.

El profesor tiene que estar consciente que la interdisciplina es una forma de abordar los complejos problemas que enfrenta la humanidad, lo cual implica un tratamiento multidimensional y que sería imposible desde disciplinas aisladas, con fronteras que limitan y fragmentan el conocimiento.

También es válido considerar que existen dos condiciones necesarias y suficientes para que el impacto de la perspectiva interdisciplinaria sea el esperado en la solución de problemas o como métodos para llegar a encontrar las respuestas que necesitan los grandes desafíos del mundo de hoy, las cuales son:

1. Dominio de las disciplinas que interactúan, por ejemplo, si se estudia la pobreza, desde el enfoque de un pedagogo, un trabajador social o un sociólogo, hay que tener las competencias disciplinares o específicas de cada una de esas profesiones.

2. Crear un "ambiente favorable", lo cual significa dejar a un lado los egos y celos profesionales, fomentar el trabajo colaborativo y cooperativo con efectividad, no ocultar información entre los expertos de las dos o más disciplinas que interactúan, donde el éxito no es de una sola disciplina, sino de todas y el mayor beneficiario sería la sociedad, en este caso ilustrativo del combate a la pobreza para lograr eliminarla o mitigar sus efectos en una región o localidad determinada.

\section{Referencias}

[1] Morin, E. (2006). El Método 1. La Naturaleza de la Naturaleza. Ediciones Cátedra.

[2] Pérez Luna, E., Alfonso Moya, $\mathrm{N} \&$ Curcu Colón, A. "Transdisciplinariedad y educación", Revista Venezolana de Educación, Vol. 17, No. 56, 2012, pp. 15-26.

[3]Tobón, S. (2013). Formación integral y competencias: Pensamiento complejo, currículo, didáctica y evaluación. Ed. 4ª . Bogotá: Ecode.

[4] Restrepo, I. "Tendencias mundiales en la gestión de recursos hídricos, desafíos para la ingeniería del agua", Revista Ingeniería y Competitividad, Vol. 6, No. 1, 2004, pp. 63-70.

[5] Díaz Barriga, F., Lule, M.; Rojas, S. \& Saad, S. Metodología de Diseño Curricular para la Educación Superior. México. Trillas, 1990. 
[6] Cerezal Mezquita, J. \& Fiallo Rodríguez, J. Los métodos científicos en las investigaciones pedagógicas. La Habana. Editorial Pueblo y Educación, 2002.

[7] Vasco, E. Objetivos específicos, indicadores de logros y competencias ¿y ahora estándares?, Educación y Cultura, Vol. 62, 2003, pp. 33-41.

[8] Zabalza, M. El Espacio Europeo de Educación Superior: Innovación en la Enseñanza Universitaria. Innovación Educativa, Vol. 18, 2008, pp. 69-95.

[9] Agazzi, E. (2007). El desafío de la interdisciplinariedad: dificultades y logros. 20 de octubre de 2017, del Departamento de Filosofía de la Universidad de Navarra Sitio web http://www.arvo.net/pdf/El\%20desafio\%20de\%interdisciplinariedad\% 2dificultades\%20y20\%logros.htm

[10] Álvarez de Zayas, C. La Escuela en la vida. (3ra Edición). La Habana. Editorial Pueblo y Educación, 1999.

[11] Fernández de Alarza, B. La interdisciplinariedad como base de una estrategia para el perfeccionamiento del diseño curricular de una carrera de ciencias técnicas y su aplicación en la Ingeniería Automática en la República de Cuba. Tesis para la Obtención del Grado Científico de Doctor en Ciencias Pedagógicas. La Habana, 2001.

[12] Ackoff, R. Rediseñando el Futuro. México D.F.: Limusa-Noriega, 2005. 